\title{
SOME MECHANISMS OF COLOR RECEPTION \\ FOUND BY ANALYZING THE ELECTRO- RETINOGRAM OF FROG
}

\author{
PART 1. COLOR CHARACTERISTICS OF THE \\ MULTIPLE OFF-RESPONSE AND NUMBER \\ OF RETINAL ELEMENTS*
}

\author{
MASAYOSI GOTO AND NAOKI TOIDA ${ }^{\dagger}$ \\ Department of Physiology, Kyûsyû University, Hukuoka, Japan
}

As Granit (6) has summarized in his book, many have observed that in electroretinograms (ERG) the off-response as well as the on-response often rise in two or more steps. This irregularity must be largely due to the differences in property between different types of retinal elements. On the off-effect of the frog's retina, Granit and Wrede (11) stated that the first fast potential was due to cone activity and the later rise to the rods. Adrian $(1,2)$ asserted that the presence of a rapid component potential is apparently due to the photopic mechanism and a slower component due to the scotopic in the various kinds of eye of warm-blooded animals, including the human. The different behaviors of rods and cones, however, do not seem to be the sole reason for the occurrence of multiple on- and off-responses, because such effects have been observed in the pure cone eyes of the chameleon, horned toad (15) and chicken (4).

Studying the discharges of well-isolated elements from the cat's retina with the microelectrode technique, Donner (3) has found that on-off-elements tend to produce their maximum effects at certain phases of the discharge varying with different colors, namely that in red an early frequency maximum is obtained, in green a somewhat later one and in blue the latest one. Motokawa and co-workers have also published a series of results of their investigations on the electrical excitability of the eyes of human (16), cat (17), frog and toad $(16,18)$ after illumination with lights of different wave-lengths. The electrical excitability becomes supernormal after cessation of the conditioning light, appearing in time order of red, green and blue, as in the preceding case. Thus the possibility may exist that the multiple on- and off-responses are phenomena in 'ERG' corresponding to those reported by Donner and by Motokawa. However, a detailed investigation of the ERG from that point of view is lacking, as Donner has pointed out.

As we reported in the preceding paper (4), a convenient method of catching the typically multiple off-responses in some definite shape was found after a systematic investigation of the natures of the multiple off-effects. The ques-

Received for publication March 20, 1954.

* This report was read before the 26th (1949) annual meeting of the Japanese Physiological Society.

$\dagger$ 後藤昌義, 問田直幹 
tion to be clarified now is whether these multiple off-waves are somehow peculiarly influenced by illumination with light of various colors.

\section{METHOD AND TECHNIQUE}

The experiments were usually carried out on the excised eyeballs of the frog. Two platinum electrodes were placed one on the cornea and the other on the hind pole of the eye, and these were connected with a direct coupled amplifier of two stages. The ERG was recorded photographically by the aid of Yokogawa's electromagnetic oscillograph with D- or F-type vibrator.

As mentioned above, it was necessary to catch the large definite-shaped and distinctly split off-deflection in order to analyze the character of each peak. These demands were met by using the following procedure. Throughout the experiment the eye was kept at a constant low temperature between about $5^{\circ}$ and $10^{\circ} \mathrm{C}$. The eye was adapted sufficiently to light of strong intensity, before the light was put out and then a light of faint intensity (0.4 lux) and short duration (about 1 second) was given repeatedly to the eye at 2 seconds' intervals by use of a metronome. A few minutes after cessation of the strong illumination, the second and third rises of off-response to the faint light began to appear with a half second or longer latency after the first fast one, and gradually grew markedly in size. This state in which the experiments were performed usually lasted over one hour. One of the white lights repeatedly given was abruptly replaced with a colored light, or one particular color was given just at the cessation of repeated white illuminations, in order to observe the deformations elicited by these procedures in the multiple and definite-shaped off-response. For light illumination a patch of opaque glass of $4-6^{\circ}$ in visual angle was placed in front of the eye and white or colored lights of various intensities were projected on it from different sources with or without grey or Wratten's color filters.

The transmission curves of the color filters were determined with the sectorphotometer, and the range of $10 \%$ transmission was found to be above 586 $\mathrm{m} \mu$ for the red filter, $490-588 \mathrm{~m} \mu$ for the green filter and 404-506 $\mathrm{m} \mu$ for the blue filter. The stimuli of different wavelengths which are physiologically equal to the rods were obtained by revising the transmission curves of the filters with the visual purple (VP) absorption curve determined by Lythgoe (14).

RESULTS

Time course of multiple off-response following color illumination of physically equal energy

The color characteristic of the multiple off-response was studied by varying the wave-length of light used for illumination. An example of the series of experiments at $7^{\circ} \mathrm{C}$ is given in figure 1 . The energy of each illuminating color was equalized by the aid of a thermopile connected to a galvanometer. The multiple off-response for white light generally appears in two or three steps. As can be seen in the figure, however, no marks of the later peaks are elicited and merely the first small peak appears unchanged in the case of red light. This fact suggests that the first fast peak is specific for red and the later one 


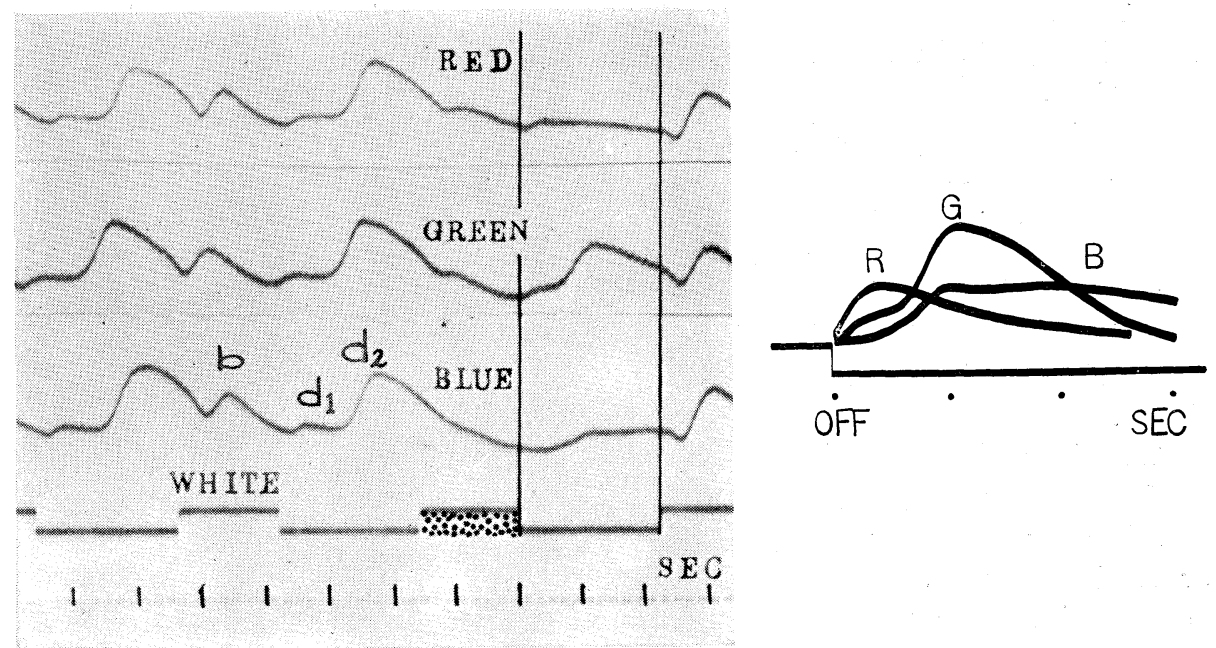

FIG. 1. Color characteristics of time course of multiple off-response obtained by using physically equal energy. Left set of curves: one case of the experiments $\left(7^{\circ} \mathrm{C}\right)$. Note deformations specific for colors, in comparison with curves by white stimuli. Right set: different time courses for different colors presented together for comparison.

for other colors than red. When the time course of off-response for green or blue light is compared in detail with that for white light or with those for the other colors, it becomes conclusive that the first peak corresponds to red, the central largest one to green and the latest slow rise to blue, although the last appears, at times separated and at times fused with the second green rise.

\section{Phenomena of selective inhibition and acceleration}

From the facts mentioned above it becomes highly probable that each wavelet of multiple off-effect is a special response to one definite color. Then, if one definite color is left unextinguished after the cessation of white light, can any selective deformation take place on the off-response? This is another problem to be solved before the character of multiple off-response can be clari-

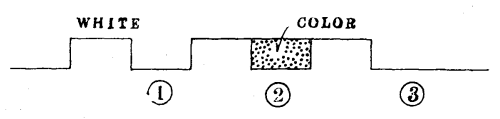

(1) CONTROL

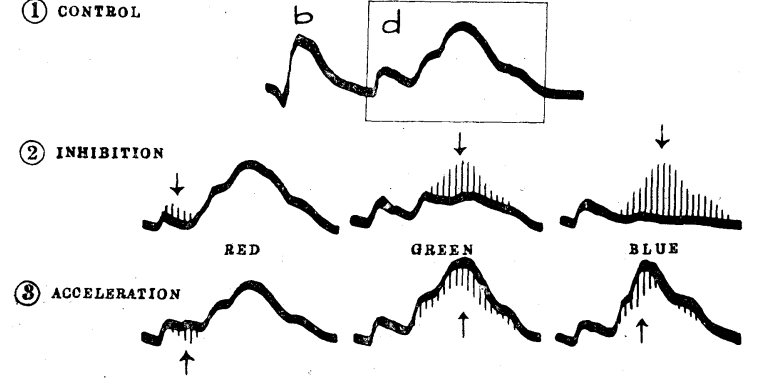

FIG. 2. Selective inhibition and post-inhibitory acceleration of multiple off-response. Procedure is shown schematically in uppermost inset, in which numbers denote the period during which each of the data below was obtained. Deformations from control (1) are shown as shades in curves of both inhibition (2) and acceleration (3). 
fied. The results of the experiment performed for this purpose are illustrated in (2) of figure 2, in which the special deformation from the control is shaded. As can be clearly seen, a special inhibition is caused by each remaining color in each time course, i.e. the first fast off-wave is inhibited by red, the third rise by green and all the later rises by blue.

Another important phenomenon is shown in (3) of figure 2. There occured a remarkable enhancement of the inhibited peaks in (2) after the cessation of the next white light.

Similar evidence of inhibition and acceleration was also obtainable by superposing a faint color illumination of long duration upon the repeating white stimuli.

The data given in this section leads to the following conclusion from the standpoint of color distribution of off-wavelets that red light is apparently responsible for the first peak, green light for the third large one and blue light for the broad rise covering all the ridge from the second to the last. It must be noted, however, that the acceleration after the inhibiting blue illumination is so marked in the second rise that it seems as though the second rise is responsible for blue, and accordingly the off-wavelets appear successively for red, 'blue' and green. If this is the case, it is inconsistent with the data of the preceding section. This question was solved unexpectedly by an experiment described in the next section.

Separation of cone activity from that of rod by using equal VP stimulations

Granit and co-workers $(9,11)$ discovered that the cone system was responsible for the early fast off-effect and the rods for the later slow rise. However, we obtained some evidence indicating that the number of multiple off-wavelets is at least 3 or more, as is stated in full detail in the next section. Then the question arises whether one or two or all of the later rises correspond to the activity of the rod system.

In order to separate the rod activity from that of the cone we used Granit's method of epual VP stimulation. When an almost dark-adapted eye is subjected to stimulation of faint light, properties of the electrical response of the eye must depend to a great extent on VP. It is known that, when the light is so ad-

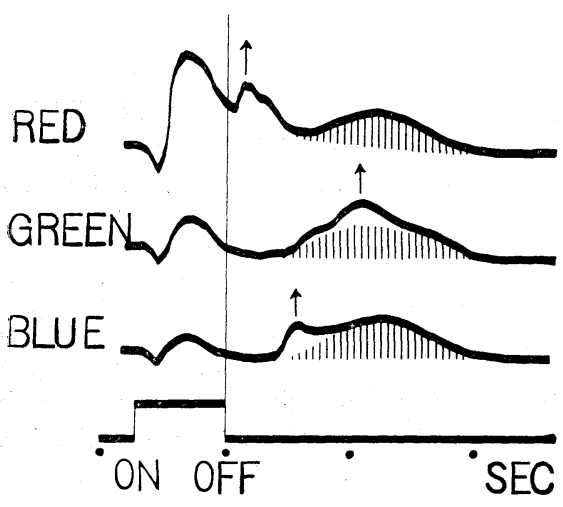
justed as to have an equal bleaching efficiency on visual purple, the off-response should be identical in size and shape in all wave-lengths if rods alone are in action, and that any difference that may occur must be due to the activity of the cone system.

FIG. 3. Time courses of frog's ERG produced by equal VP stimulations of red, green and blue light. Part of off-response common to all colors is shaded, showing rod activity. Arrows denote specific off-responses of the cone system to definite color. 
The results obtained are shown in figure 3. For red light the first and the fourth rises appear remarkably far separatad, while for green the third and the fourth waves, piled up together, make a relatively large deflection with a long latent time, and for blue the second and the fourth rises are partly gathered together to compose a flat plateau. Thus the fourth peak always appears in all kinds of color illumination of equal VP stimuli. This fact indicates that the fourth rise of off-response is responsible for the activity of the rod system and accordingly all other faster rises, i.e. the first red, the second blue and the third green are reduced to the cone system.

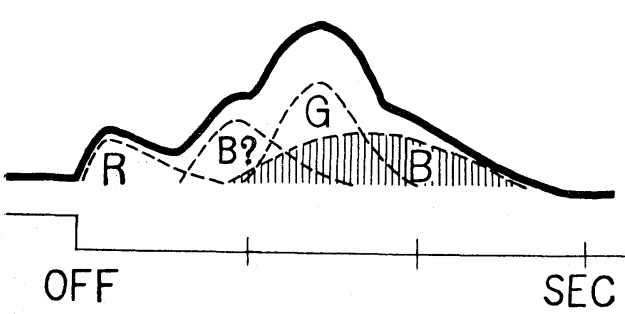

FIG. 4. Analysis of a multiple offresponse of a frog's ERG. Continuous curve: standard time course for white. Broken curves: probable responses of retinal elements for red $(R)$, blue $(B$ ?), green $(G)$ and blue $(B)$ respectively, in which shaded rise for blue is ascribable to rod activity and the other three to cone system.

In figure 4 all the above-stated results are summarized and the estimated construction of the multiple off-response is illustrated in a scheme. It must be noted that for the blue part of the spectrum two elevations appear in the response: one is the second rise due to the cone system and the other the fourth rise due to the rod. This fact seems to explain the somewhat queer phenomena in which for the blue light there appeared irregularly the second rise in one case, and the fourth rise or summation of both rises in another case. It is, however, still a question why the second rise appears for the blue light. This shoud be solved by further study.

Number and shape of multiple off-responses

In our preceding paper (4) we reaffirmed the views of several authors that the multiple responses are quite different in character from the rhythmical oscillations originating in the synchronized impulses of the optic nerve. In this section merely the multiple off-response inherent in ERG will be described, without paying any attention to the rhythmical oscillation in order to avoid confusion.

As illustrated in figure 4, the multiple off-response generally appears as three main split rises, as mentioned repeatedly in our other reports $(4,19)$, but in subsequent experiments we occasionally met with a further multiplication, each one of these main rises manifesting itself in two or more small peaks. Several examples of these phenomena are given in figure 5 , in which the small wavelets of off-responses were denoted in dots. Thus it may be evident that the total number of multiple off-wavelets consists of at least six or more.

In figure 5 the intensity of stimulating white lights becomes gradually fainter from $a$ to $c$ in declining order, as is clearly seen from the size of the on-response also. It will be noted that the fainter the intensity of stimulating light, the smaller is the first main rise and, inversely, the larger the later rise. 
This phenomenon will coincide with the view that the faster rise is responsible for the cone system and the later one for rods.

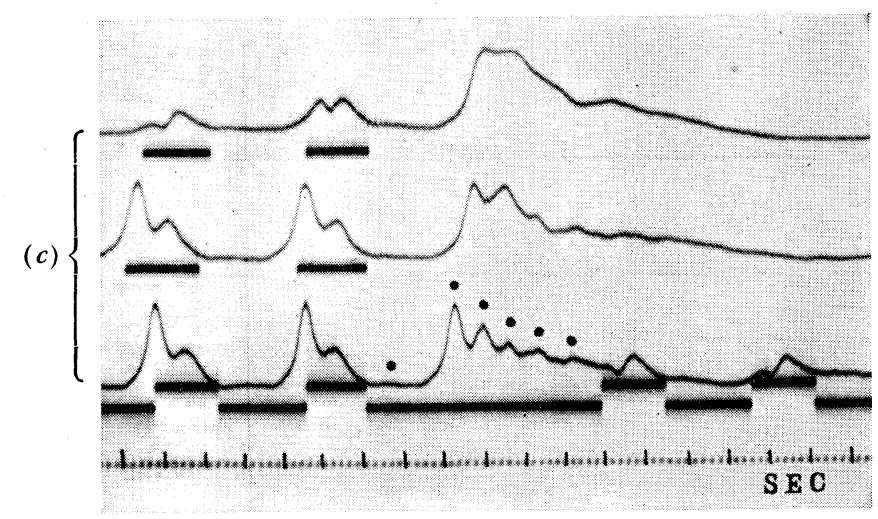

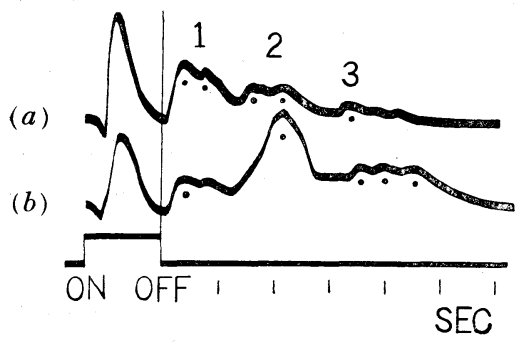

FIG. 5. Highly multiple off-responses appearing during a long interval after repeated test illuminations. Note number, shape and latency of peaks. The last peak sometimes has a surprisingly long latency (four seconds or longer). Further explanations are given in the text.

\section{DISCUSSION}

On ERG of the frog's eye the multiple response generally appears far more freqently and also distinctly in the off-effect than in the on-effect. Why this difference occurs is an interesting and important problem. In 1938 Hartline (12) was successful in obtaining the records of action potential from a single optic fiber of the frog. He found that about half the nerve fibers were "on-off," about one-third were "off" and the remaining fibers were "on". It was also found by Granit and Tansley (10) that the off-elements were more sensible to light of a longer wave-length (larger Red/Blue Ratio) than the on-elements. Granit $(7,8)$ concluded by several criteria that the pure on-elements were reduced to rods. As to the off and on-responses of the on-off elements which are most sensible to red light among these three kinds of elements, the former is elicited by red light more readily than the latter. Thus in a twofold sense it is highly probable that in the frog's retina the on-effect is responsible for rods for the most part, while the off-response is more concerned with cone activity, which usually produces several modulator curves. This may be in accord with our result that the off-response shows a tendency to split more apparently than the on-response.

Donner (3) studied the frequency of discharge from well-isolated elements of a dark-adapted cat's retina and found that at the onset or cessation of mono- 
chromatic lights with physically equal energy or of equal VP stimulus, many of the on-off-elements tended to produce their maximum effects at certain phases of the discharge depending upon the color, while the pure on-elements did not show such a tendency. His results mentioned above can be well interpreted by the difference between on- and off-responses of multiple ERG and by the color dependence of multiple off-response, although, of course, the frequency maximum of the discharge in the cat is attained within a far shorter time than the crest of the response in a cold-blooded animal like the frog.

It is also of interest to know which layer in the retina contributes to the splitting and then to the specific patterns of the response to color. Donner (3) discussed this in his report and from several standpoints stated that some sort of nervous interactions or synaptic properties in the retina would be more probably responsible for the definite patterns of optic nerve discharge to color than the receptors themselves. According to Granit, the ERG consists of three component potentials of different origins, $P_{I}, P_{I I}$ and $P_{I I I}$; but in our cases the effect of $P_{I}$ may safely be disregarded, because the stimulation used in the experiment was so short and so faint that no sign of $P_{I}$ appeared. Hence, as to a cause of the splitting, $\mathrm{P}_{I I}$ and $\mathrm{P}_{I I I}$ components will have to be considered. As mentioned above, the multiple response appears far more distinctly in $d$-wave which is produced by the interaction between $\mathrm{P}_{I I}$ and $\mathrm{P}_{I I I}$ than in $b$-wave which is apparently dependent on $\mathrm{P}_{I I}$. $\mathrm{P}_{\mathrm{III}}$ will play a larger rôle in composing the $d$ wave than $P_{I I}$, especially in such a typical I-retina as the frog's eye. Therefore it may be considered that $P_{I I I}$ is the component potential which is mainly responsible for the splitting of off-response. Particularly from the fact that in our preceding report (4) with chicken the off-wave splits distinctly even when the positive $\mathrm{P}_{\mathrm{II}}$ component almost disappears under the experimental condition of low temperature, this view seems to gain support.

\section{SUMMARY}

The character of each wavelet in the multiple off-response was investigated in ERG of isolated frog's eyes at a definite low temperature, by using color illuminations of physically equal energy and of equal bleaching efficiency on visual purple. The results obtained were as follows.

A series of multiple off-wavelets due to white-light was found to contain patterns of specific response to colors-red, blue, green and blue in time order - of which the last blue rise was due to the activity of rods and all other earlier rises to the cone system.

Similar results were obtained in experiments on selective adaptation to definite colors so that a particular rise in the multiple off-response was inhibited by adaptation to a definite color and the inhibited peak contrarily, appeared markedly enhanced after removal of the adapted light.

Each main rise for any of the three original colors in the multiple offresponse occasionally appeared as a group of smaller peaks. Thus the total number of multiple off-wavelets inherent in ERG may be at least six or more, suggesting the presence of so many kinds of retinal elements in the frog's eye. 
The nature and origin of multiple ERG were discussed in reference to several authoritative works on the subject.

A part of expenses of this research was defrayed by a grant from the Ministry of Education.

\section{REFERENCES}

1. ADRIAN, E. D. The electric response of the human eye. J. Physiol. 104: 48-104, 1945.

2. ADRIAN, E. D. Rod and cone components in the electric response of the eye. J. Physiol. $105: 24-37,1946$.

3. DONNER, K. O. The spike frequencies of mammalian retinal elements as a function of wave-length of light. Acta Physiol. Scand. 21 : Supplementum 72: 1-59, 1950.

4. Goto, M. AND ToidA, N. About the multiple $d$-wave in electroretinogram. Jap. J. Physiol. 4 ; 123-30, 1954.

5. Granit, R. Colour receptors of the frog's retina. Acta Physiol. Scand. 3 : 137-51, 1941.

6. GRANIT, R. Sensory mechanisms of the retina. Oxford Univ. Press, 1947.

7. GRANIT, R. Neural organization of the retinal elements, as revealed by polarization. J. Neurophysiol. $10: 239-52,1947$.

8. Granit, R. Physiology of vision. Ann. Rev. Physiol. 12: 485-502, 1950.

9. GRANIT, R. AND MUNSTERHJELM, A. The electrical responses of darkadapted frog's eyes to monochromatic stimuli. J. Physiol. 88: 436-58, 1937.

10. GRANIT. R. AND TANSLEY, K. Rods, cones and the localization of preexcitatory inhibition in the mammalian retina. J. Physiol. $107: 54-66,1948$.

11. GRANIT, R. AND WREDE, C. M. The electrical responses of light-adapted frog's eyes to monochromatic stimuli. J. Physiol. 89 : 239-56, 1937.

12. HARTLine, H. K. The response of single optic nerve fiber of the vertebrate eye to illumination of the retina. Amer. J. Physiol. 121: 400-15, 1938.

13. HARTRIDGE, H. Recent advances in the Physiology of vision. London: Churchill, 1950.

14. LYThgOE, R. J. The absorption spectra of visual purple and of indicator yellow. $J$. Physiol. 89 : 331-58, 1937.

15. Meservey, A. B. AND Chaffee, E. L. Electrical response of the retina in different types of cold-blooded animals. J. opt. Soc. Amer. $15: 311-30,1937$.

16. MотокAWA, K. Retinal processes and their role in color vision. J. Neurophysiol. 12 : 291-303, 1949.

17. Motokawa, K., IWAma, K. AND EBE, M. Retinal color processes in cats. Jap. J. Physiol. 2 : 198-207, 1952.

18. MOTOKAWA, K., IWAMA, K. AND TUKAhARA, S. Color processes in single retinal elements. Tohoku J. exp. Med. 53 : 399-406, 1951.

19. ToIDA, N. AND Goto, M. On a new clue in electroretinogram for research of color reception. Medicine and Biology (Japanese) 29: 72-75, 1953. 